\title{
A percepção de valor dos planos diretores: um estudo exploratório ${ }^{1}$
}

\section{The perception of value of master plans: an exploratory study}

\author{
Santos, João Pablo'; Miron, Luciana I. G.2; \\ I UFRGS, Pinhalzinho - SC, Brasil - arq.joaopablo@gmail.com \\ 2UFRGS - luciana.miron@ufrgs.br
}

\begin{abstract}
RESUMO
Recentemente, a percepção de valor vem sendo utilizada para entender a relação do usuário com o ambiente construído. Muitos desses estudos tem utilizado a técnica laddering, a qual busca revelar os valores pessoais dos usuários, questões não conscientes e que implicam diretamente na aquisição de produtos. Contudo, no planejamento urbano, estudos dessa natureza ainda são poucos. Portanto, o objetivo deste artigo é testar o uso da técnica para explicitar a percepção de valor de cidadãos e técnicos sobre um Plano Diretor. O estudo foi estruturado a partir de revisão de literatura e da aplicação da técnica, cuja coleta de dados é realizada através de entrevistas em profundidade. As entrevistas foram conduzidas com 7 cidadãos e 1 técnico de uma prefeitura. A partir dessas entrevistas, foram estruturados Mapas de Hierarquia de Valor (MHV) para explicitar a percepção dos entrevistados. Foi identificado que os moradores tem como limitação a falta de conhecimento específico sobre o Plano em si, porém levam em consideração atributos mais concretos do ambiente construído, tais como a infraestrutura urbana e aos equipamentos públicos. Já os técnicos, apresentaram uma profundidade maior nas respostas e atribuem maior valor ao Plano.
\end{abstract}

Palavras-chave: Percepção de valor, laddering, plano diretor.

\begin{abstract}
Recently, the perception of value has been used to understand the relationship of the user with the built environment. Many of these studies have used the technique laddering, which seeks to reveal the personal values of users, nonconscious issues and that directly implicate in the acquisition of products. However, in urban planning, studies of this nature are still very few. Therefore, the objective of this article is to test the use of the technique to explain the value perception of citizens and technicians about a Master Plan. The study was structured based on literature review and the application of the technique, whose data collection is performed through in-depth interviews. The interviews were conducted with 7 citizens and 1 technician from a city hall. From these interviews, Value Hierarchy Maps (MHV) were structured to explain the perception of the interviewees. Residents were identified as having a lack of specific knowledge about the Plan itself, but took into account more concrete attributes of the built environment, such as urban infrastructure and public facilities. Already the technicians, presented a greater depth in the answers and attributed greater value to the Plan.
\end{abstract}

\footnotetext{
${ }^{1}$ SANTOS, João Pablo; MIRON, Luciana I. G. A Percepção de Valor dos Planos Diretores: Um Estudo Exploratório. In: II SIMPÓSIO NACIONAL DE GESTÃO E ENGENHARIA URBANA: SINGEURB, 2019, São Paulo. Anais... Porto Alegre: ANTAC, 2019.
} 
Keywords: Perception of value, laddering, master plan.

\section{INTRODUÇÃO}

A necessidade de se prever ou antecipar impactos e mudanças no ambiente urbano é de suma importância para promover o equilíbrio na cidade, onde a infraestrutura existente possa ser capaz de absorver certos equipamentos e/ou adensamentos populacionais propostos (KRAFTA, 2006). Este planejamento é regido nas cidades brasileiras pelos Planos Diretores, o qual Nygaard (2005, p. 25) defende que pode ser "(...)considerado como o instrumento mais completo que o Estado já teve à sua disposição para interferir na organização e no controle do espaço urbano". Sua aplicação efetiva demanda esforços de diferentes esferas do Estado, envolvendo também a população local. O documento ainda engloba diversos aspectos de manejo da cidade, controlando as diferentes ações dos agentes urbanos tendo como foco o futuro das cidades e o bem-estar comum da população (SOUZA, 2012).

Ao abordar a percepção de valor para com o plano diretor neste estudo, o mesmo será interpretado aqui pela sua relação com os aspectos comportamentais dos públicos envolvidos. O termo "valor" como um julgamento, pode distinguir-se do termo "valores" - as crenças individuais, morais e ideais fundamentais (THOMSON et al., 2003). O conceito de percepção de valor, nesse contexto, pode ser influenciado pelos valores pessoais ou individuais (IKEDA; VELUDO DE OLIVEIRA, 2005, p.3). Ikeda e Veludo de Oliveira (2005), explicam que há subjetividade no conceito de valor, o qual pode expresso por "gostar ou não gostar". Trata-se de um julgamento subjetivo, dependendo dos valores, do interesse do indivíduo e de sua individualidade.

Thomson et al. (2003) também explicam que o valor é dependente de tempo e que pode mudar. Assim, considera-se aqui que o valor é subjetivo e dependente do tempo e do contexto. Neste sentido temporal, é possível fazer uma aproximação com a abordagem do Plano Diretor, pois quando é colocado em ação, pode causar efeitos positivos e/ou negativos, e o tempo é algo crucial para analisar o impacto desta ação, a partir daí obtendo a percepção de valor.

Holbrook (2006) ainda conceitua o valor percebido como extrínseco, o qual é considerado o valor de algo (meio) que leva a um fim. Ou seja, se alguém valoriza um objeto por si só, o mesmo não é necessariamente valioso na percepção deste usuário, pois pode partir de uma avaliação subjetiva do sujeito e que não está vinculado ao objeto em si, mas ao que o objeto o leva a ter como produto final desejado (HOLBROOK, 2006).

A ideia de explorar qual a percepção do consumidor com o produto (que consome) originouse na teoria de cadeias meios-fim de Gutman (1982). Segundo Veludo de Oliveira e Ikeda (2008), a teoria visa compreender como os valores pessoais dos consumidores influenciam em suas escolhas, estabelecendo uma associação entre as características de um produto que está sendo usado/consumido/comprado, ou seja, os "meios" são os produtos ou atividades nas quais as pessoas estão envolvidas (A ou C) e o "fim" é o valor, ou estado valorado de existência (V). Esses elementos estão encadeados de tal forma que os "meios" levam a um "fim", ou seja, os atributos dos produtos (A) levam a consequências ou benefícios decorrentes do uso do produto (C) que, por sua vez, ajudam o indivíduo a alcançar estados ou valores pessoais (V) (GUTMAN, 1982).

O modelo adotado neste artigo, baseado em Gutman (1982) foi proposto por Olso e Reynolds (1983) com o seguinte desdobramento para a cadeia meios-fim: (a) atributos, (b) consequências funcionais, (c) consequências psicossociais, (d) valores instrumentais e (e) valores terminais, somando assim seis níveis de hierarquia de valor.

A técnica laddering tem sido usualmente utilizada para a construção de um mapa de hierarquia de valor, o qual explicita a cadeia meios-fim. "(...) é um interessante instrumento de descoberta dos constructos formadores de valor para o usuário e dos valores pessoais não conscientes que os entrevistados revelam a partir de sucessivos questionamentos." (IKEDA et al., 2014, p.65). A técnica é aplicada segundo lkeda et al. (2014) por meio de entrevistas individuais em profundidade que traduzem os atributos que levam aos valores pessoais dos 
entrevistados e sua motivação a adquirir certos produtos, tal como a cadeia meios-fim aborda, a fim de passar do nível mais concreto de percepção para algo mais profundo e abstrato.

Nesta pesquisa considera-se que o Plano Diretor tem valor instrumental considerado como um "meio" para se obter um fim. A técnica laddering foi selecionada por ser adequada para a explicitação da percepção desde os elementos mais concretos aos mais abstratos. Sendo assim, artigo tem como objetivo testar o uso da técnica laddering para explicitar a percepção de valor de cidadãos e técnicos sobre um Plano Diretor. Pretende-se com isso, explorar a possibilidade de analisar o valor atribuído ao plano diretor, visando compreender o que a população entende por serem os benefícios decorrentes deste instrumento de planejamento.

\section{MÉTODO}

Foram realizadas 8 entrevistas em profundidade ao total. Dessas entrevistas, 7 foram com cidadãos sobre suas percepções sobre um plano diretor. Para possibilitar uma comparação, também foi entrevistado 1 técnico de prefeitura, arquiteto e urbanista., A partir dos resultados das entrevistas foram feitos mapas de hierarquia de valor (MHV). Esses mapas de hierarquia de valor, segundo Ikeda et al. (2014) são representações gráficas e esquemáticas das associações feitas pelos entrevistados durante as entrevistas.

O mapeamento neste estudo foi feito manualmente, ou seja, elaborado sem o auxílio de softwares de laddering, em função do estudo ser considerado exploratório nesta temática.

\section{MHV PLANO DIRETOR - TÉCNICO ARQUITETO E URBANISTA}

Para avaliação do plano diretor, primeiramente foi desenvolvido um MHV específico a partir da visão de um técnico arquiteto e urbanista. Esse MHV foi estruturado conforme os atributos do Plano, consequências do uso e os objetivos esperados pelo técnico com a aplicação efetiva deste.

Alguns aspectos do atributo concreto Plano Diretor foram considerados mais importantes que outros (representados com linhas mais espessas), tal como as questões de habitação, acesso a infraestruturas básicas, postos de saúde e comércio por exemplo e também a questão de zoneamentos adequados ao contexto da área. Foi observado que o técnico por possuir maior domínio e conhecimento para com o Plano Diretor, apresentou questões muito mais profundas as quais julgou necessárias para que um bom planejamento pudesse ser feito na cidade, estas percepções foram estruturadas no Mapa de Hierarquia de Valor abaixo (Figura 01). Cabe considerar que esse mapeamento foi feito com apenas um técnico, por tratar-se de um estudo exploratório. 
Figura 01 - MHV esperado com base na percepção de técnico arquiteto e urbanista

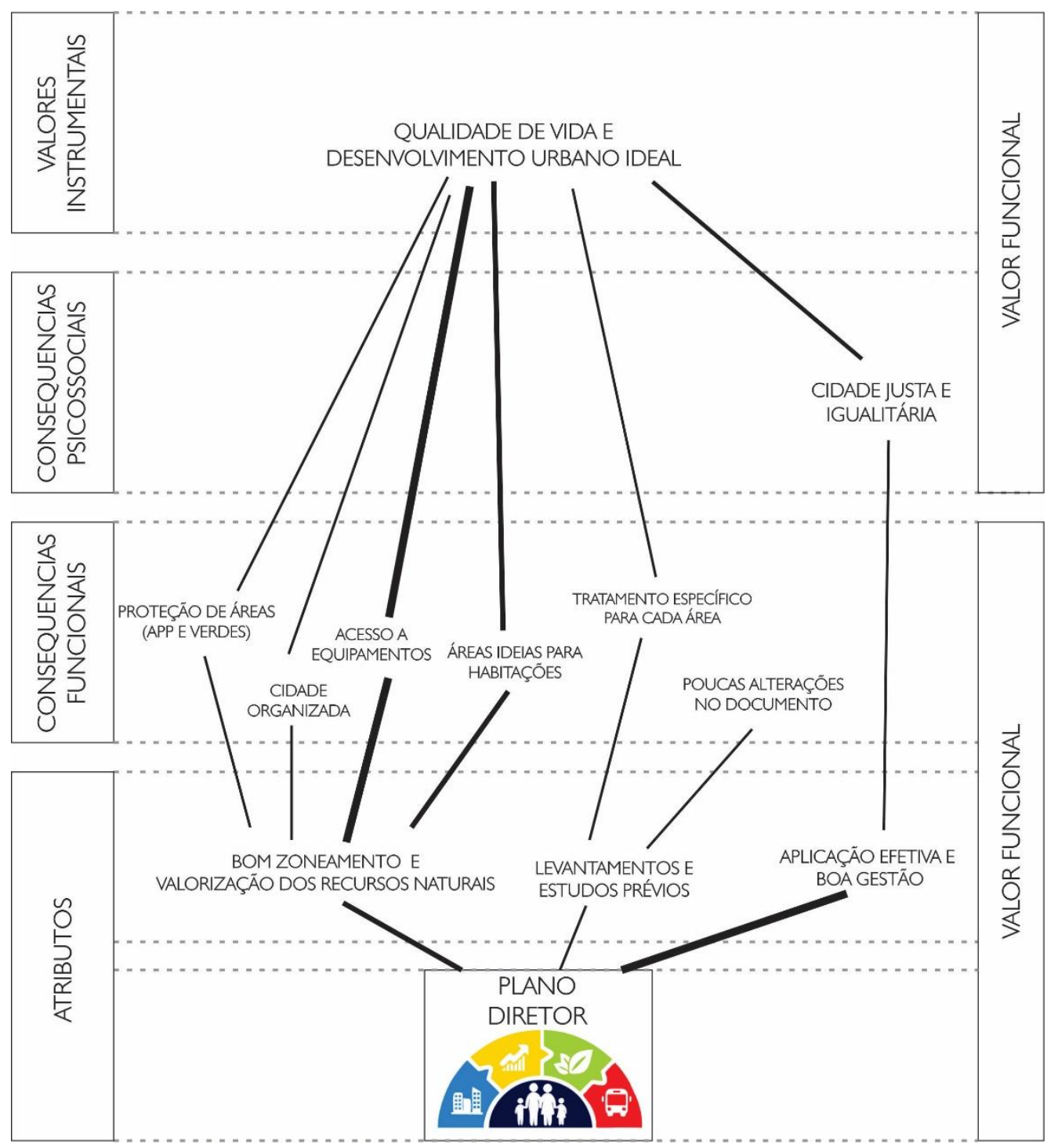

Fonte: Os autores (2018)

\section{MHV PLANO DIRETOR - MORADORES}

Por meio da técnica laddering e do mapeamento feito de forma manual, foi possível estruturar - mapeamento de valor percebido pelos cidadãos, sendo que sete pessoas foram entrevistadas para que houvesse uma amostragem mínima para análise.

O questionário tinha como objetivo entender o que os moradores esperam e acreditam que este instrumento de planejamento deva proporcionar a suas vidas e a cidade. Como foi possível perceber no mapeamento (Figura 02), os cidadãos apontaram a infraestrutura básica como principal benefício esperado, esse fato se deve devido ao pouco conhecimento por parte dos entrevistados sobre o que realmente é/significa o Plano Diretor para sua cidade. As questões sobre o envolvimento dos indivíduos em audiências públicas para tomadas de decisões em prol da cidade se mostraram uma surpresa para a maioria, que não tinha conhecimento de que poderiam participar de tal forma na elaboração do documento. 
Figura 02 - MHV esperado com base na percepção dos cidadãos

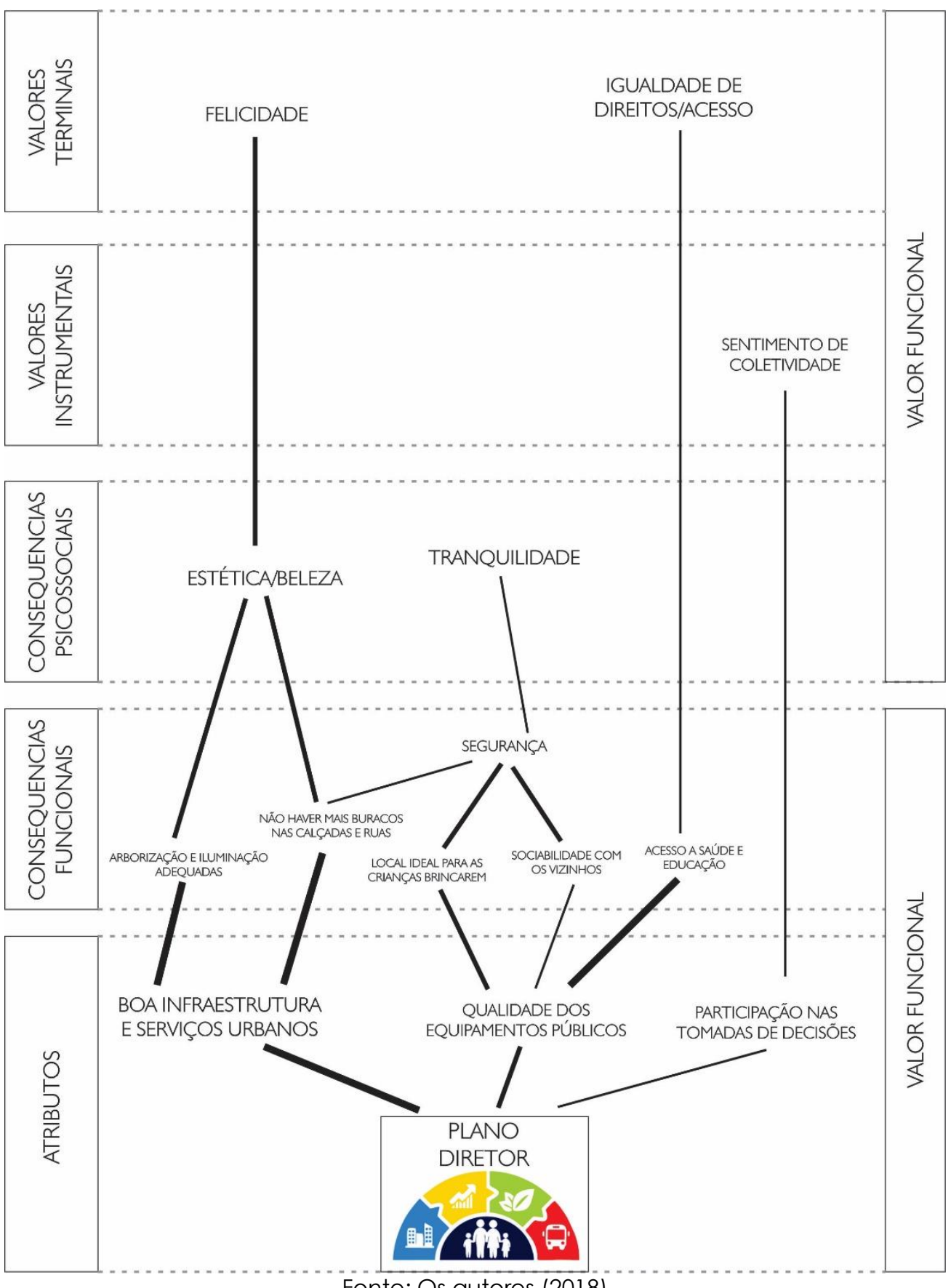

\section{CONSIDERAÇÕES FINAIS}

O uso e adaptação dos conceitos e teorias sobre a percepção de valor para com algo que não é verdadeiramente um "produto" de compra em si, porém um instrumento imposto/proposto aos cidadãos, mostrou-se pertinente no tocante à compreensão desta percepção por um outro olhar, demonstrando ser possível estudos dessa natureza. A percepção de valor e a importância do Plano Diretor pelos entrevistados contrasta de maneira significativa.

As cadeias geradas pelos dois mapeamentos divergem em alguns pontos, sendo possível compreender que o técnico possui sua visão específica e acadêmica, compreendendo um maior número de implicações e considerando as prioridades para o desenvolvimento de uma cidade. Os cidadãos explicitaram percepções individuais e de natureza imediata, visível, como por exemplo na cadeia "plano diretor - boa infraestrutura e serviços urbanos - 
arborização e iluminação adequadas - estética e beleza - felicidade", a qual representa as cadeias mais fortes do mapa. Essa cadeia demonstra o nível de entendimento e de conhecimento por parte dos cidadãos para com o plano diretor e, aparentemente, estão relacionadas às características mais concretas, mais visíveis aos olhos, mais relacionadas ao embelezamento o lugar. Características, que quando resolvidas de maneira adequada, trazem o sentimento de felicidade "por morarem em um local bem iluminado e arborizado", assim citado por eles.

Outros pontos que foram citados pelo técnico também apareceram na cadeia do MHV dos moradores, como por exemplo a "qualidade de equipamentos públicos" e o acesso aos mesmos, sendo que foram levantadas principalmente questões como a falta de postos de saúde próximos e praças que atendam a demanda local. Essa percepção individual é fruto do meio em que essas pessoas vivem e presenciam a cidade acontecendo, cada uma em seu contexto. É possível então corroborar com a ideia de Harvey (1980, p.36) sobre o espaço urbano no contexto abordado neste estudo, onde o mesmo "(...) é visto como multidimensional, não homogêneo, talvez descontínuo, altamente personificado, e significativo de diferentes modos, em diferentes contextos de atividade social."

O MHV dos cidadãos demonstrou pouco aprofundamento, dificultando o real objetivo da técnica. $\bigcirc$ uso da laddering propõe entrevistas em profundidade, envolvendo questões mais específicas e profundas. Porém, os entrevistados (na maioria moradores leigos), não possuíam um domínio sobre o que realmente era o Plano Diretor, por se tratar de uma ferramenta de planejamento complexa para entendimento. Isso dificultou o avanço das entrevistas, que deveriam priorizar uma abstração muito maior.

A contribuição deste estudo se deu no sentido de testar uma técnica, utilizada em outros estudos que relacionam o ambiente construído à percepção de usuários, em um contexto distinto, o qual busca relacionar a percepção de cidadãos com um instrumento de planejamento da cidade. A partir da aplicação desta, foi possível fazer um esboço inicial das possibilidades desse tipo de estudo. Além disso, foi possível um delineamento inicial sobre o papel do planejamento urbano para a comunidade, salientando a grande importância que a participação pública desempenha. A partir desses resultados exploratórios, sugere-se como estudos futuros a investigação sobre:

- como a laddering poderia auxiliar na melhoria da relação entre os técnicos que elaboram o plano e os cidadãos, buscando explorar processos mais participativos baseados na percepção da população;

- a realização de entrevistas com representantes de bairros que geralmente participam de audiências de Planos Diretores, os quais levam as demandas do público;

- o uso da técnica como base para auxílio na tomada de decisão técnica, a partir da percepção da população.

\section{AGRADECIMENTOS}

O presente trabalho foi realizado com apoio da Coordenação de Aperfeiçoamento de Pessoal de Nível Superior - Brasil (CAPES).

\section{REFERÊNCIAS}

GUTMAN, J. A means-end chain model based on consumer categorization processes. Journal of Marketing, v. 46, p. 60-72, 1982.

HARVEY, David. A Justiça Social e a Cidade. 1 ed. São Paulo: Hucitec, 1980. 
HOLBROOK, M.B. Consumption experience, custumer value, and subjective personal introspection: An illustrative photograpic essay. Journal of Business Research. V. 59. p. 714725, 2006.

IKEDA, Ana Akemi; CAMPOMAR, Marcos Cortez; CHAMIE, Beatriz Cavalcante. LADDERING: Revelando a coleta e interpretação dos dados. REMark-Revista Brasileira de Marketing. 2014. Disponível em: http://www.revistabrasileiramarketing.org/ojs2.2.4/index.php/remark/article/view/2726/pdf_1 75

KRAFTA, Romulo. Cidades Versus Planos Diretores. In: PANIZZI, Wrana (Org.). Outra Vez Porto Alegre.1 ed. Porto Alegre: Cirkula, 2006.

MONTEIRO, Deyvid Alex de Bitencourt. Proposta de um método para avaliação da percepção de valor em empreendimentos habitacionais de interesse social. Dissertação de Mestrado (Planejamento Urbano e Regional) - Escola de Arquitetura, Universidade Federal do Rio Grande do Sul, Porto Alegre, 2015.

NYGAARD, Paul Dieter. Planos Diretores de Cidades: discutindo sua base doutrinária. $1^{\mathrm{a}}$ ed. Porto Alegre-RS. UFRGS Editora, 2005.

SOUZA, Marcelo Lopes de. Mudar a Cidade: Uma Introdução Crítica ao Planejamento e à Gestão Urbanos. $11^{a}$ Ed. Rio de Janeiro: Bertrand Brasil, 2011.

THOMSON, D.S.; AUSTIN, S.A.; DEVIN-WRIGHT, H.; MILLS, G.R. Managing value and quality in design. Journal of Building Research and Information, v. 31, p. 334-345, 2003. 